Original Article

\title{
A randomized controlled trial of the computer-based cognitive rehabilitation program for children (CoTras-C) to examine cognitive function and visual perception in children with developmental disabilities
}

\author{
Jin-Hyuck Park, OT, MPH ${ }^{1)}$, Ji-Hyuk Park, OT, $\mathrm{PhD}^{1)^{*}}$ \\ 1) Department of Occupational Therapy, College of Health Science, Yonsei University: 1 Yonseidae-gil, \\ Wonju, Gangwon-do 220-710, Republic of Korea
}

\begin{abstract}
Purpose] This study aimed to investigate the effects of a computer-based cognitive rehabilitation program for children with developmental disabilities. [Subjects] Subjects included 29 children with developmental disabilities. [Methods] The subjects were randomly allocated to either the experimental group or control group. Experimental group subjects received computer-based cognitive rehabilitation using the CoTras- $\mathrm{C}$ while control group subjects received conventional cognitive rehabilitation. All subjects received 20 sessions (2 days a week for 10 weeks) of the experimental or control intervention for 30 minutes. To compare the two groups, the KoreanDevelopmental Test of Visual Perception-2 and Kaufman Assessment Battery for Children were performed before and after the intervention. [Results] Both groups showed statistically significant improvement in their scores after intervention. Additionally, there were significant differences in the scores between the two groups. [Conclusion] The computer-based cognitive rehabilitation with CoTras-C may be helpful in improving the recovery of cognitive function and visual perception in children with developmental disabilities.

Key words: Computer-based cognitive rehabilitation, Cognitive function, Developmental disabilities, Visual perception
\end{abstract}

(This article was submitted Jul. 29, 2015, and was accepted Aug. 30, 2015)

\section{INTRODUCTION}

Developmental disabilities (DD) are a chronic delay in development, including conditions such as cerebral palsy, intellectual development disorder, autism, and mixed type DD. It is thus used as a comprehensive term for a variety of diagnoses ${ }^{1)}$. Children with DD have problems with physical, cognitive, social, sensory, and communication skills ${ }^{2}$. These unique problems have a negative impact on the lives of their family as well as of the children with $\mathrm{DD}^{1)}$.

Among the problems faced by children with DD, impairment in cognitive function and visual perception causes problems in processing environmental stimuli, which makes it difficult for them to adapt to their environment and live in independently ${ }^{2}$. After entering elementary school, due to an increase in demanding activities of daily living, problems caused by impairments of cognitive function and visual perception become more evident ${ }^{3}$. As compared to prior

Corresponding author. Ji-Hyuk Park (E-mail: otscientist@ yonsei.ac.kr)

(C2015 The Society of Physical Therapy Science. Published by IPEC Inc. This is an open-access article distributed under the terms of the Creative Commons Attribution Non-Commercial No Derivatives (by-ncnd) License $<$ http://creativecommons.org/licenses/by-nc-nd/3.0/> . to school entry, school-aged children are required to complete tasks within a limited time at school or home and are expected to exhibit sophistication and organization in their activities $^{4}$.

The literature indicates that there are intervention methods to improve cognitive function and visual perception in children with $\mathrm{DD}$, such as attention training programs, sensory integration, and tabletop activities ${ }^{5,6}$. These methods are remedial approaches to restore and facilitate the improvement of cognitive function and visual perception caused by $\mathrm{DD}^{7}$.

Although various conventional treatment methods for children with DD have been applied in clinical settings, the controversies over the most effective protocol continues ${ }^{8}$. In order to compensate for these problems, recently computerbased cognitive rehabilitation (CBCR) has been used in clinical settings ${ }^{8)}$. Several studies concluded that $\mathrm{CBCR}$ was an effective treatment for recovery of cognitive function and visual perception ${ }^{9}$. However, most such studies recruited adults or the elderly with stroke, traumatic brain injury, or dementia $^{10)}$. Few studies have investigated the effects of $\mathrm{CBCR}$ on cognitive function and visual perception in children with DD. Additionally, the existing CBCR programs have predominantly targeted adults, and there is a lack of CBCR programs for children ${ }^{8}$.

In 2011, Kim developed a CBCR program for children 
called CoTras- $\mathrm{C}^{8)}$. However, there is still insufficient evidence for the clinical effectiveness of this program. Therefore, there is a need to investigate the effectiveness of the CoTras-C using objective methods.

Accordingly, in this study, we conducted a randomized controlled trial to investigate the effectiveness of the CBCR program for children with reference to their cognitive function and visual perception.

\section{SUBJECTS AND METHODS}

Children with DD were recruited from a local rehabilitation hospital. We screened the participants based on the inclusion criteria used in previous studies on CBCR for children $^{8,11)}$. The inclusion criteria for participation were as follows: (i) DD diagnosed by a pediatrician or rehabilitation physician; (ii) children with DD, exhibiting similar symptoms (children with limited cognitive function and visual perception); (iii) presence of DD diagnosed by performance on the Korean-Developmental Test of Visual Perception-2 (K-DTVP-2); (iv) a below normal score on the Kaufman Assessment Battery for Children (K-ABC); (v) ability to use the controller with the upper extremity without spasticity; and (vi) ability to understand instructions within three repeated explanations. Parents signed the informed consent form before commencement of the study, according to the code of ethics of the World Medical Association (Declaration of Helsinki, version 2004).

Two clinical measurements were administered on participants. The first was the K-DTVP-2, which comprises 8 sub-tests (eye-hand coordination, position in space, copying, figure-ground, spatial relations, visual closure, visual-motor speed, and form constancy). This test can be used to measure visual perception in children aged 4 to 8 years, and helps to confirm the extent of visual perception disability according to developmental stages ${ }^{12}$. It provides an index of motor-reduced visual perception (MRP) and visual motor integration (VMI) and combines the two indices to interpret the overall level of visual perception (General Visual Perception; GVP) ${ }^{13)}$. The second measure was the K-ABC, designed for use with children aged 2.5 to 12.5 years. It assesses the intelligence quotient and achievement, and entails 2 subscales, the achievement scale (ACH) and mental processing composite (MPC), and 16 sub-tests. The expected mean raw score is 100 , with a standard deviation of $15^{14}$ ) The number of sub-tests implemented changes depending on the age of the children tested. Therefore, in this study, we used the sub-tests, depending on the age of the subjects. All clinical measurements were administered before and after the intervention by a blinded occupational therapist with 4 years' experience. After the baseline session, eligible participants were randomly allocated to either the control group (CG) or experimental group (EG) by a research assistant who was not involved in the intervention.

During the intervention sessions, the EG participants received 20 sessions ( 2 days a week for 10 weeks) of CBCR with the CoTras-C program (Netblue Co., Ltd, Korea). The CoTras-C consists of about 4,000 game-based training task on skills including attention, memory, visuo-motor organization, coordination, and others. The CoTras- $\mathrm{C}$ with a charac-
Table 1. Subjects' characteristics

\begin{tabular}{llcc}
\hline & & $\begin{array}{c}\text { EG } \\
(\mathrm{n}=15)\end{array}$ & $\begin{array}{c}\text { CG } \\
(\mathrm{n}=14)\end{array}$ \\
\hline \multirow{2}{*}{ Gender } & Male & 9 & 10 \\
\multirow{2}{*}{ Age (months) } & Female & 6 & 4 \\
Education & Preschool & $69.73 \pm 8.33$ & $71.93 \pm 8.07$ \\
& School & 10 & 10 \\
Main & Mother & 11 & 4 \\
care-giver & Grandmother & 4 & 8 \\
\hline
\end{tabular}

Mean \pm standard deviation. CG: Control Group; EG: Experimental Group

ter design and voice suitable for children, makes the training easy by using the touch monitor and CoTras-C controller. The difficulty level of all the tasks can be modified to suit the individual participants' abilities. Training data are automatically stored for accuracy analysis, which helps develop an appropriate treatment plan ${ }^{8,11)}$. In this study, the EG participants received the visual perception training that comprised spatial relations, spatial memory, concentration, eye-hand coordination, eye movement, and figure-ground perception. The duration of intervention for the CG participants was matched to that of the EG participants. The CG participants received a conventional cognitive rehabilitation that focused on visual perception, using pencil and paper activities. All participants received either the EG or CG intervention for 30 minutes per a session.

Data analyses were performed using the SPSS program version 20.0. The $\chi^{2}$ test and independent t-test were used to compare the differences in the general characteristics of the two groups. The independent t-test was used to compare the between group means and changes in values, while the paired t-test was used to test the differences in the continuous variables within the groups. Statistical significance was accepted for values with $\mathrm{p}<0.05$.

\section{RESULTS}

The 29 participants who fulfilled the inclusion criteria were randomly allocated to the two groups as follows: 15 to the EG and 14 to the CG. All participants' characteristics have been summarized in Table 1.

After intervention, the participants in both the groups showed a significant increase in their scores on the KDTVP-2, ACH of the K-ABC, and MPC of the K-ABC ( $\mathrm{p}$ $<0.001$ ) (Tables 2 and 3). Additionally, there were significant differences in the changes in the K-DTVP-2, ACH of the $\mathrm{K}-\mathrm{ABC}$, and MPC of the K-ABC between the two groups ( $\mathrm{p}$ $<0.001$ ) (Table 4).

\section{DISCUSSION}

Although studies on the effectiveness of the CBCR program for children are lacking ${ }^{8,11)}$, it is increasingly used in clinical settings because of its advantages ${ }^{15}$ ). Therefore, the present study investigated the effectiveness of the CBCR 
Table 2. Subjects' K-DTVP-2 scores before and after the intervention

\begin{tabular}{lcc}
\hline & \multicolumn{2}{c}{ K-DTVP-2 $(\mathrm{GVP})$} \\
\cline { 2 - 3 } & Pre-test & Post-test \\
\hline EG & 66.80 & $79.87^{* *}$ \\
$(\mathrm{n}=15)$ & $(3.63)$ & $(2.92)$ \\
$\mathrm{CG}$ & 67.79 & $76.43^{* *}$ \\
$(\mathrm{n}=14)$ & $(2.78)$ & $(3.90)$ \\
\hline
\end{tabular}

$* \mathrm{p}<0.05, * * \mathrm{p}<0.001$

Mean (standard deviation). CG: Control Group; EG: Experimental Group; GVP: General Visual Perception; K-DTVP-2: Korean-Developmental Test of Visual Perception-2

Table 4. Comparison of change in K-DTVP-2 and K$\mathrm{ABC}$ scores

\begin{tabular}{lcc}
\hline & $\begin{array}{c}\text { EG } \\
(\mathrm{n}=15)\end{array}$ & $\begin{array}{c}\text { CG } \\
(\mathrm{n}=14)\end{array}$ \\
\hline K-DTVP-2 & 13.07 & $8.64^{* *}$ \\
(GVP) & $(1.44)$ & $(3.15)$ \\
K-ABC & & \\
& 45.93 & $22.79^{* *}$ \\
ACH & $(4.23)$ & $(2.75)$ \\
& 6.90 & $4.18^{* *}$ \\
MPC & $(0.71)$ & $(0.80)$ \\
\hline
\end{tabular}

$* \mathrm{p}<0.05, * * \mathrm{p}<0.001$.

Mean (standard deviation). ACH: Achievement Scale; CG: Control Group; EG: Experimental Group; GVP: General Visual Perception; K-ABC: Kaufman Assessment Battery for Children; K-DTVP-2: Korean-Developmental Test of Visual Perception-2; MPC: Mental Processing Composite

program with reference to cognitive function and visual perception in children with DD. The results of this study indicated that the CBCR program for children may improve cognitive function and visual perception in children with DD. The clinical improvement in the K-DTVP-2 and K-ABC scores was greater in the $\mathrm{EG}$ after intervention, as compared to that in $\mathrm{CG}$ participants, confirming that $\mathrm{CBCR}$ is a more effective intervention for children with $\mathrm{DD}$ as compared to conventional intervention.

Most CBCR programs used in Korea were developed for adults and these effectiveness has been confirmed by several studies. However, in some parts of CBCR program for adults, the stimuli presented on the screen are not suitable for children and do not sustain interest in them. Therefore, it is difficult to use such programs with children ${ }^{8}$. To resolve these problems, Kim developed a CBCR program for children called CoTras- $\mathrm{C}^{8)}$.

The effectiveness of CBCR with the CoTras-C on cognitive function and visual perception have been proved in several studies ${ }^{8,11)}$. The use of a computer program helps in the cognitive development of children by facilitating their intellectual ability, stimulating curiosity, and allowing for creative thinking ${ }^{16)}$. In 2014, Park indicated that children with DD who received CBCR with the CoTras-C showed in-
Table 3. Subjects' K-ABC scores before and after the interventions

\begin{tabular}{lcccc}
\hline & \multicolumn{2}{c}{ ACH } & \multicolumn{2}{c}{ MPC } \\
\cline { 2 - 5 } & Pre-test & Post-test & Pre-test & Post-test \\
\hline EG & 494.20 & $540.13^{* *}$ & 68.60 & $75.56^{* *}$ \\
$(\mathrm{n}=15)$ & $(25.46)$ & $(23.91)$ & $(3.74)$ & $(4.09)$ \\
CG & 508.64 & $531.42^{* *}$ & 70.25 & $74.42^{* *}$ \\
$(\mathrm{n}=14)$ & $(17.99)$ & $(16.99)$ & $(3.18)$ & $(3.26)$ \\
\hline
\end{tabular}

$* \mathrm{p}<0.05, * * \mathrm{p}<0.001$.

Mean (standard deviation). ACH: Achievement Scale; CG: Control Group; EG: Experimental Group; MPC: Mental Processing Composite

creased K-DTVP-2 and K-ABC scores, which is consistent with the results of the present study ${ }^{11)}$. Visual perception is the ability to interpret and understand the environment by processing information that is contained in visible light. Visual perception is closely related to cognitive function, and therefore, an improvement in visual perception has been found to have a positive impact on cognitive function ${ }^{13)}$, which was also confirmed in the results of the present study.

This study conclude that CBCR with the CoTras-C can be used as a rehabilitation approach to improve cognitive function and visual perception in children with $\mathrm{DD}$, which suggests that the CoTras-C may be used as an alternative to other existing CBCR programs.

Although the sample size employed in the present study was small, the findings of this study are significant because CBCR is a novel approach that broadens the scope of clinic based intervention to home-based ones. Additionally, children living in a modern society have several opportunities to receive this treatment ${ }^{17}$.

The present study did not examine the effectiveness of CBCR with the CoTras-C on the activities of daily living of children with DD. Therefore, in future, studies with a large sample size are needed to investigate the clinical effectiveness of the CoTras- $\mathrm{C}$ as a rehabilitation program for activities of daily living as well as cognitive function and visual perception.

\section{REFERENCES}

1) Cho HJ, Lee MJ: A study on the concepts of developmental disabilities. J Dev Disabil, 2012, 16: 1-21.

2) Bottcher SA: Cognitive retraining. A nursing approach to rehabilitation of the brain injured. Nurs Clin North Am, 1989, 24: 193-208. [Medline]

3) Dankert HL, Davies PL, Gavin WJ: Occupational therapy effects on visual-motor skills in preschool children. Am J Occup Ther, 2003, 57: 542-549. [Medline] [CrossRef]

4) Kim JM, Song SJ: Analysis of Korean children's visual perceptual development using TVPS-R. J Emot Behav Disord, 2007, 23: 241-262.

5) Case-Smith J, Miller H: Occupational therapy with children with pervasive developmental disorders. Am J Occup Ther, 1999, 53: 506-513. [Medline] [CrossRef]

6) Watling R, Deitz J, Kanny EM, et al.: Current practice of occupational therapy for children with autism. Am J Occup Ther, 1999, 53: 498-505. [Medline] [CrossRef]

7) Schaaf RC, Miller LJ: Novel therapies for developmental disabilities: occupational therapy using a sensory integrative approach. Ment Retard Dev Disabil Res Rev, 2005, 11: 107-109. [CrossRef]

8) Kim YG, Lee MJ: The effect on computer-based cognitive rehabilitation program for children (CoTras-C) for the cognitive ability and visual per- 
ception in developmental disability. J Rehabil Res, 2013, 17: 391-414.

9) Kim YG: The effects of Korean computer-based cognitive rehabilitation program (CoTras) for the cognition and ADL in stroke. J Korean Soci Occup Ther, 2011, 19: 75-88.

10) Kim YG: The effects on computer-based cognitive rehabilitation program (CoTras) for the visual perception in brain injury. J Rehabil Res, 2012, 16 401-409.

11) Park SW: The effect of the computer-based cognitive rehabilitation program on visual perception, cognition, and activities of daily living for school-aged children with developmental disabilities. Unpublished doctoral dissertation, Daegu University, 2014.

12) Moon SB, Yeo GW, Cho YT: Korean-Developmental Test of Visual Perception. Seoul: Hakjisa, 2003.
13) Hammil DD, Pearson NA, Voress JK: Developmental test of visual perception, 2nd ed. Texas: Pro-ed, 1993

14) Kaufman AS, Kaufman NL: K-ABC interpretation manual. MN: American Guidance Service, 1984.

15) Cho HY, Kim KT, Jung JH: Effects of computer assisted cognitive rehabilitation on brain wave, memory and attention of stroke patients: a randomized control trial. J Phys Ther Sci, 2015, 27: 1029-1032. [Medline] [CrossRef]

16) Clements DH: Enhancement of creativity in computer environments. Am Educ Res J, 1991, 28: 173-187. [CrossRef]

17) Cha YJ, Kim H: Effect of computer-based cognitive rehabilitation (CBCR) for people with stroke: a systematic review and meta-analysis. NeuroRehabilitation, 2013, 32: 359-368. [Medline] 